\title{
Multi-directional emission and detection of spin waves propagating in yttrium iron garnet with wavelengths down to about $100 \mathrm{~nm}$
}

\author{
Stefan Maendl, a) and Dirk Grundler², b) \\ 1) Lehrstuhl für Physik funktionaler Schichtsysteme, Physik Department E10, \\ Technische Universität München, James-Franck-Str. 1, 85748 Garching, \\ Germany \\ ${ }^{2)}$ Laboratory of Nanoscale Magnetic Materials and Magnonics, \\ Institute of Materials (IMX) and Institute of Microengineering (IMT), \\ École Polytechnique Fédérale de Lausanne (EPFL), 1015 Lausanne, \\ Switzerland
}

(Dated: 17 April 2018)

We performed broadband spin-wave spectroscopy on $200 \mathrm{~nm}$ thick yttrium iron garnet containing arrays of partially embedded magnetic nanodisks. Using integrated coplanar waveguides (CPWs) we studied the excitation and transmission of spin waves depending on the presence of nanomagnet arrays of different lateral extensions. By means of the grating coupler effect we excited spin waves propagating in multiple lateral directions with wavelengths down to $111 \mathrm{~nm}$. They exhibited group velocities of up to $1 \mathrm{~km} / \mathrm{s}$. Detection of such short-wavelength spin waves was possible only in symmetrically designed emitter/detector configurations, not with a bare CPW. We report spin waves propagating between grating couplers under oblique angles exhibiting a wave vector component parallel to the CPW. The effective propagation distance amounted to about $80 \mu \mathrm{m}$. Such transmission signals were not addressed before and substantiate the versatility of the grating coupler effect for implementing nanomagnonic circuits.

The complementary metal oxide semiconductor technology might face technological shortcomings in the future in that miniaturization slows down due to increased power dissipation ${ }^{1}$. At the same time, very large scale integration densities make interconnections challenging $^{2}$. Collective spin excitations (spin waves) in magnetic materials have entered the international technology roadmap for semiconductors with the prospect of interconnections with low power consumption and wave-based data processing. Following Khitun et al. ${ }^{1}$ the functional throughput scales with the inverse wavelength cubed, and spin waves (SWs) with deep-submicron wavelengths are required. Furthermore a large group velocity $v_{\mathrm{g}}$ and large propagation length are needed ${ }^{1}$. Thin films prepared from the ferrimagnetic insulator yttrium iron garnet $\mathrm{Y}_{3} \mathrm{Fe}_{5} \mathrm{O}_{12}$ (YIG) ${ }^{3-10}$ are expected to provide relevant characteristics and enable size-reduced magnonic holography memories, spin wave interferometers and cellular nonlinear networks ${ }^{1,11-16}$. However, high-power parametric pumping of shortwavelength SWs in $\mathrm{YIG}^{17}$ counteracts low-power consumption, and a SW emitter based on a spin transfer torque nanopillar ${ }^{18}$ is not compatible with the insulating ferrimagnet. SW emission from a bound pair of stacked spin vortices formed in antiferromagnetically coupled multilayers ${ }^{19}$ has not yet been realized in YIG either. Recently Yu et al. used the magnonic grating coupler (MGC) effect ${ }^{20}$ and demonstrated transmission of SWs with a wavelength $\lambda$ of $88 \mathrm{~nm}$ in $20 \mathrm{~nm}$ thick $\mathrm{YIG}^{21}$. The SWs were excited by coplanar waveguides (CPWs) fabricated on top of a magnetic nanodisk array. They propagated in YIG in a direction perpendicular to the CPW. In integrated magnonic circuits propagation in multiple directions is needed however.

In this work we report the excitation and phase-sensitive detection of spin waves propagating along different lateral directions between two parallel MGC-based emitters and detectors. Our devices incorporated nanodisk arrays of different lateral extension (compare

\footnotetext{
a) stefan.maendl@ph.tum.de

b) dirk.grundler@epfl.ch
} 


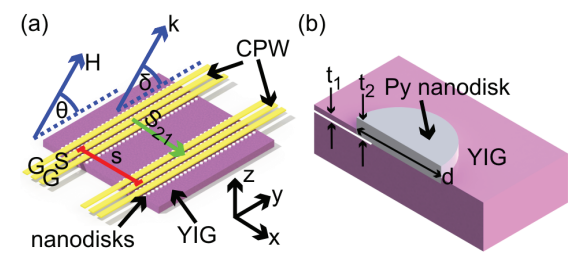

FIG. 1. Sketch of a symmetrically designed SW emitter and detector device that hosts nanomagnet arrays (white disks) directly underneath two CPWs (yellow stripes). The sketch represents sample MGC01. Spin wave propagation in the YIG film of thickness $t$ is detected via scattering parameter S21. The center-to-center distance of CPWs amounts to $s$. The external magnetic field is applied in the sample plane under an angle $\theta$. The angle $\delta$ defines the emission angle of SWs with in-plane wave vector k. A bare CPW emits SWs with $\delta=90^{\circ}$. (b) Cross section of a sample near a recessed nanodisk. The white line indicates the etching depth.

sketches in Fig. 5 in which white disks illustrate the arrays). We demonstrate detection of SWs with $\lambda$ down to $111 \mathrm{~nm}$ which propagated over about $80 \mu \mathrm{m}$ under oblique angles with respect to the relevant CPWs. Multi-directional emission and detection of propagating SWs is crucial for multi-branched nanomagnonic circuits ${ }^{13}$.

Our experiments were based on a commercially available YIG film of thickness $t=200 \mathrm{~nm}$ grown by liquid phase epitaxy ${ }^{5,22}$ on a 3" (111) GGG wafer ${ }^{23}$. The YIG film was studied in Ref. [9] and exhibited a small damping of $\alpha=1.0 \times 10^{-4}$. The nanostructured nanodisks were fabricated using electron beam lithography of a resist mask containing holes, argon ion milling of YIG through the holes and physical vapor deposition of permalloy $\left(\mathrm{Ni}_{80} \mathrm{Fe}_{20}\right)$ at room temperature before lift-off processing. We made use of the process presented in Ref. [24] for creation of a bicomponent magnonic crystal and further exploited in Ref. [20] for grating couplers. The permalloy nanodisks arranged on a square lattice were thus partially embedded in YIG [Fig. 1 (b)]. The parameter $t_{1}$ denotes the etching depth used to embed nanodisks. It was nearly identical for all samples. The periodicity $p$ was $700 \mathrm{~nm}$. We report on arrays covering different areas of the YIG (compare sketches in Fig. 5). The geometrical parameters of the arrays are summarized in Tab. I. To excite spin-processional motion we use two integrated coplanar waveguides (CPW) (Fig. 1) connected to a multi-port vector network analyzer (VNA) ${ }^{25,26}$. Real and imaginary parts of scattering parameters $S i j$ with $i, j=1,2$ were measured, reflecting the phase of detected spin waves. The separation of the CPWs was $s=60 \mu \mathrm{m}$. The width $w$ for signal (S) and ground $(\mathrm{G})$ lines was $2.1 \pm 0.2 \mu \mathrm{m}$. The edge-to-edge distance $r$ of signal and ground lines was $1.4 \pm 0.2 \mu \mathrm{m}$. The CPWs were $380 \mu \mathrm{m}$ long and consisted of a $4.5 \mathrm{~nm}$ thick chromium and $120 \mathrm{~nm}$ thick gold layer. The CPWs show a maximum excitation amplitude at $k_{\mathrm{I}}=0.9 \mathrm{rad} / \mu \mathrm{m}$ with $\delta=90^{\circ}$. An external magnetic field $\mathbf{H}$ was applied and rotated in the sample plane. We evaluated both linear magnitude and imaginary parts from datasets $S i j$. To reduce field-independent noise we display differences $\Delta S i j$ between spectra taken at successive magnetic fields $\mathbf{H}$ or successive angles $\theta$. For line plots showing the imaginary part we subtracted a reference spectrum taken at $0 \mathrm{mT}$ and thereby explored $\mathrm{SW}$ propagation characteristics. To model field-dependent SW resonance frequencies and extract relevant in-plane wave vectors $\mathbf{k}$ from the spectra we model the measured data using the spin-wave dispersion relations introduced by Kalinikos and Slavin ${ }^{27}$. The following parameters were considered: saturation magnetization $M_{\mathrm{S}}=140 \mathrm{kA} / \mathrm{m}$ and exchange constant $A=3.32 \times 10^{-12} \mathrm{~J} / \mathrm{m}$ consistent with literature $\mathrm{e}^{6,9,28,29}$. For the wave vectors we assumed $\mathbf{k}= \pm \mathbf{k}_{\mathrm{I}} \pm \mathbf{G}_{a b}$ where $\mathbf{G}_{a b}=(a 2 \pi / p, b 2 \pi / p)$ was an in-plane reciprocal lattice vector provided by the two-dimensional nanomagnet array via the grating coupler effect ${ }^{20,21}$ and $a, b=0,1,2, \ldots$ (Fig. 2). We evaluated the wavelength $\lambda=2 \pi /|\mathbf{k}|$, the effective propagation distance $s_{\text {eff }}=\frac{s}{\sin (\delta)}$ and the SW group velocity $v_{\mathrm{g}}$ of MGC modes as discussed below.

In Fig. 3 (a) and (b) we present color-coded spectra $\Delta S 22$ and $\Delta S 21$. Figure 3 (a) displays angle-dependent spectra obtained on sample MGC01 for which the magnetic field was fixed at $70 \mathrm{mT}$. The spectra contain numerous additional SW modes that we did not resolve in angle-dependent spectra taken on a YIG film utilizing CPWs without nanomagnet arrays 


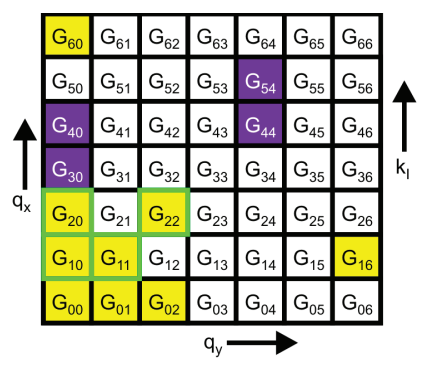

FIG. 2. Reciprocal lattice vectors $\mathbf{G}_{a b}$ in the $q_{x}, q_{y}$ plane of the square lattice. MGC modes consistent with $\mathbf{k}=\mathbf{k}_{\mathrm{I}} \pm \mathbf{G}_{a b}$ and highlighted in yellow color have been identified unambiguously in the angle scan measurement S22 of Fig. 3 (a). Wave vector $k_{\mathrm{I}}$ is collinear with the $q_{x}$ axis. A green box indicates that $|\mathbf{k}|=\left|\mathbf{k}_{\mathrm{I}} \pm \mathbf{G}_{a b}\right|$ comes close to a wave vector enabled by a high-indexed Fourier component of the radiofrequency field of the CPWs. MGC modes that are resolved in propagation signal S21 [Fig. 3 (b)] are highlighted in purple color. For consistency we define $G_{00}$ with $\left|\mathbf{G}_{00}\right|=0 \mathrm{rad} / \mu \mathrm{m}$ in this work.

\begin{tabular}{|c|c|c|c|c|}
\hline label & $t_{1}(\mathrm{~nm})$ & $t_{2}(\mathrm{~nm})$ & $d(\mathrm{~nm})$ & area $\left(\mu \mathrm{m}^{2}\right)$ \\
\hline \hline MGC01 & $4 \pm 2$ & $15 \pm 4$ & $380 \pm 20$ & $365 \times 15 *$ \\
\hline MGC02 & $6 \pm 2$ & $16 \pm 4$ & $360 \pm 20$ & $365 \times 85$ \\
\hline MGC03 & $6 \pm 2$ & $16 \pm 4$ & $360 \pm 20$ & $300 \times 65$ \\
\hline MGC04 & $6 \pm 2$ & $16 \pm 4$ & $360 \pm 20$ & $365 \times 15$ \\
\hline
\end{tabular}

TABLE I. Geometric parameters of four samples. (* sample MGC01 contains two separate nanodisk arrays of the given area). The parameter $t_{1}$ denotes the etching depth for the integration of the nanodisks, $t_{2}$ assigns the permalloy thickness and $d$ the diameter of the nanodisks.

(not shown). More than twenty additional modes are present over a frequency range from 3 to $9 \mathrm{GHz}$. We attribute the additional resonances to resonant ${ }^{21}$ and non-resonant ${ }^{20}$ magnonic grating coupler modes reflecting $\mathbf{k}= \pm \mathbf{k}_{\mathrm{I}} \pm \mathbf{G}_{a b}$. The MGC modes for which we identified $\mathbf{k}$ using the approach of $\mathrm{Yu}$ et al. ${ }^{20}$ are summarized in Tab. II.

In Fig. 3 (b) and (c) we display field-dependent SW propagation measurement $S 21$ setting $\theta$ at 0 deg. We detect several SWs transmitted between the two CPWs used, and summarize them in Tab. III. Their relevant emission angle $\delta$ (defined in Fig. 1) and effective propagation length $s_{\text {eff }}$ may vary from mode to mode. SWs with $\delta=0 \operatorname{deg}(90 \mathrm{deg})$ propagate along (perpendicular to) a CPW. From

$$
v_{\mathrm{g}}=\delta f \times s_{\mathrm{eff}}
$$

we calculate the corresponding group velocities using $\delta f$ as defined in Fig. 3 (c). Values $v_{\mathrm{g}}$, $\delta$ and $s_{\text {eff }}$ of four modes are listed in Tab. III. These SWs exhibit wavelengths $\lambda$ between $110 \mathrm{~nm}$ and $230 \mathrm{~nm}$. The group velocities are found to increase from about $500 \mathrm{~m} / \mathrm{s}$ to $1000 \mathrm{~m} / \mathrm{s}$ with decreasing SW wavelength. This trend is consistent with the theoretically calculated group velocities $v_{\text {gt }}{ }^{27}$ (Tab. III). Note that velocities $v_{\text {gt }}$ are derived assuming the empty lattice model. In the real sample, nanodisks of finite lateral dimensions are present that we expect to modify the band structure of the YIG which is underneath ${ }^{30,31}$. We attribute the discrepancies between the absolute values of $v_{\mathrm{g}}$ and $v_{\mathrm{gt}}$ to the influence of the nanomagnets. The effective propagation distance varies between $60 \mu \mathrm{m}$ and $86 \mu \mathrm{m}$ depending on the angle $\delta$. Different effective distances were not relevant for propagation signals of MGC modes in Refs. $\left.{ }^{20,21}\right]$. There transmission signals were analyzed for only $\delta=90$ deg, i.e., the 10 direction in reciprocal space of Fig. 2. In Tab. III we report properties of spin waves with $\delta \neq 90 \mathrm{deg}$ as well.

So far we analyzed sample MGC01 incorporating two nanomagnet arrays as depicted in Fig. 1 (a). In Fig. 5 we now summarize spectra $S 11$ and $S 21$ obtained on $200 \mathrm{~nm}$ thick YIG films with four different nanomagnet configurations [Fig. 5 (a) and (d)]. We display 

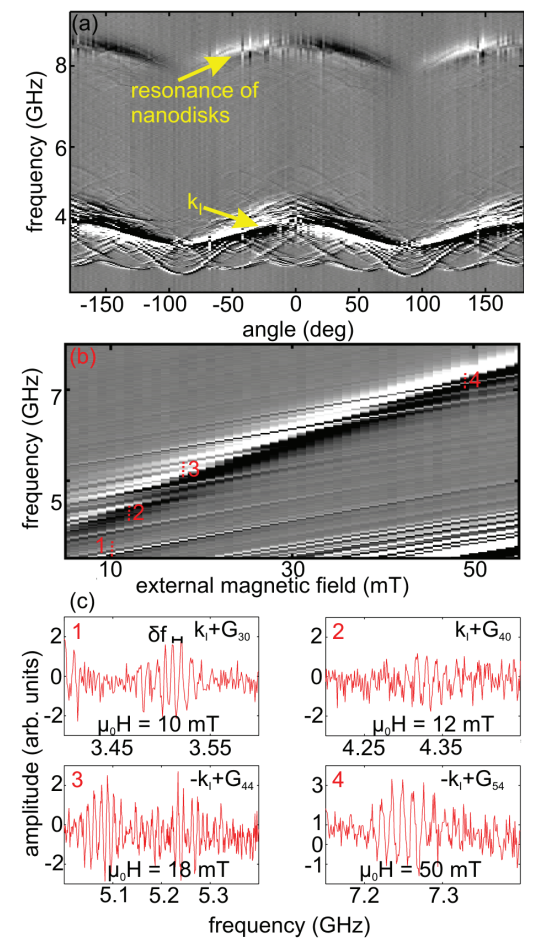

FIG. 3. Gray-color plots of (a) linear magnitude $\Delta S 22$ measured for a fixed field of $70 \mathrm{mT}$ at different angle $\theta$. (b) Imaginary part $\Delta S 21$ measured at $\theta=0 \operatorname{deg}$ as a function of $H$. The field-dependent contrast variations indicate spin-wave propagation. The contrast variations reflect oscillations in the imaginary parts $S 21$ as better visible in the spectra (line plots) shown in (c). In (c), we display spectra at four different fields at which we attribute the oscillations to different wave vectors $\mathbf{k}= \pm \mathbf{k}_{\mathrm{I}}+\mathbf{G}_{a b}$. The four fields are indicated by vertical lines and labels 1 to 4 in (b) and (c). The microwave power applied to the sample MGC01 amounted to $-10 \mathrm{dBm}$.

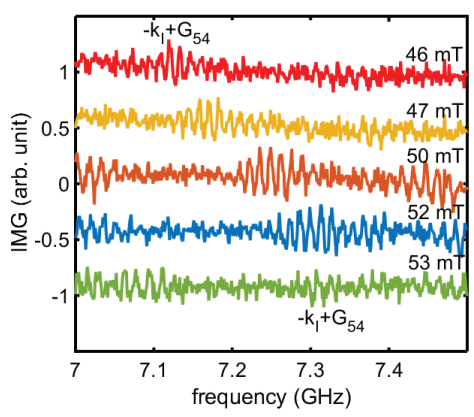

FIG. 4. Spectra (imaginary part of $S 21$ ) highlighting the field-dependent amplitude variation of the propagating spin wave corresponding to $\mathbf{k}=-\mathbf{k}_{\mathrm{I}}+\mathbf{G}_{54}$. Its wavelength amounts to $111 \mathrm{~nm}$. The amplitude increases with $H$ between 46 and $50 \mathrm{mT}$ as spin precessional motions in the nanodisk and YIG become degenerate in frequency near $50 \mathrm{mT}$ [see also Fig. 3 (c)]. At $53 \mathrm{mT}$ the amplitude is found to be small again, indicating that frequencies of spin precession in nanodisks and YIG differ.

\begin{tabular}{|l|l|l|c|}
\hline$k_{\mathrm{I}} \pm G_{10}$ & $k_{\mathrm{I}} \pm G_{01}$ & $k_{\mathrm{I}} \pm G_{20}$ & $k_{\mathrm{I}} \pm G_{02}$ \\
\hline$k_{\mathrm{I}} \pm G_{30}$ & $k_{\mathrm{I}} \pm G_{11}$ & $k_{\mathrm{I}} \pm G_{40}$ & $k_{\mathrm{I}} \pm G_{22}$ \\
\hline$k_{\mathrm{I}}+G_{60}$ & $k_{\mathrm{I}}+G_{16}$ & $-k_{\mathrm{I}}+G_{54}$ & $-k_{\mathrm{I}}+G_{44}$ \\
\hline
\end{tabular}

TABLE II. Twenty different MGC modes that were unambiguously identified in the spectra summarized in Fig. 3 (a). 


\begin{tabular}{|c|c|c|c|c|c|}
\hline MGC mode & $\lambda(\mathrm{nm})$ & $v_{\mathrm{g}}(\mathrm{m} / \mathrm{s})$ & $v_{\mathrm{gt}}(\mathrm{m} / \mathrm{s})$ & $\delta(\mathrm{deg})$ & $s_{\text {eff }}(\mu \mathrm{m})$ \\
\hline \hline$k_{\mathrm{I}}+G_{30}$ & 226 & $510 \pm 30$ & 431 & 90 & 60 \\
\hline$k_{\mathrm{I}}+G_{40}$ & 171 & $840 \pm 60$ & 630 & 90 & 60 \\
\hline$-k_{\mathrm{I}}+G_{44}$ & 125 & $988 \pm 43$ & 840 & 44 & 86 \\
\hline$-k_{\mathrm{I}}+G_{54}$ & 111 & $1046 \pm 39$ & 961 & 51 & 77 \\
\hline
\end{tabular}

TABLE III. MGC modes observed in propagation measurement of Fig. 3 (b). The table summarizes the SW wavelengths $\lambda$, experimentally and theoretically determined group velocities $v_{\mathrm{g}}$ and $v_{\mathrm{gt}}$, respectively, the emission angle $\delta$ and the effective propagation distance $s_{\text {eff }}$ of the SW. Theoretical values of the group velocity $v_{\text {gt }}$ are obtained with the formalism of Kalinikos and Slavin ${ }^{27}$ assuming the empty lattice model $^{32}$. Spin waves in the two bottom lines exhibit wave vector components parallel to the CPWs.

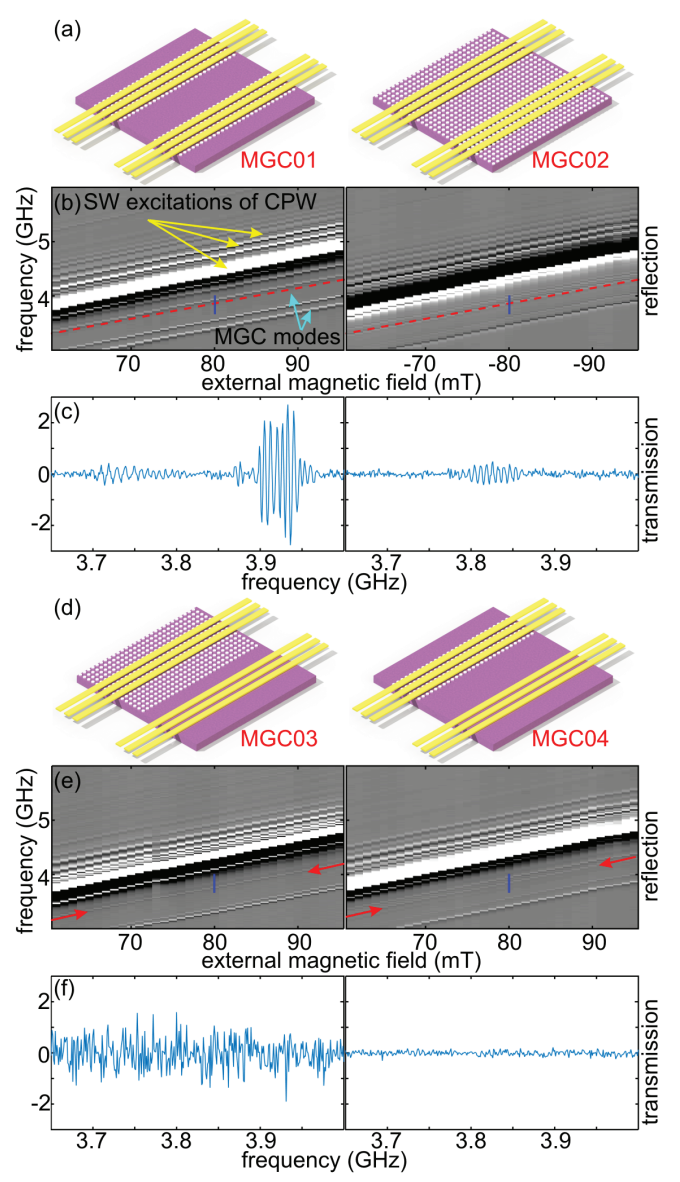

FIG. 5. (a) Sketches of samples MGC01 and MGC02 (same color code as in Fig. 1) in which we detect a mode consistent with a wave vector $\mathbf{k}_{\mathrm{I}}+\mathbf{G}_{\mathbf{1 2}}$ in both (b) reflection $(\Delta S 11$, linear magnitude) and (c) transmission signals ( $\Delta S 21$, imaginary part). In (b) the dashed red lines indicate the calculated eigenfrequencies (branch) of a $S W$ with $\mathbf{k}_{\mathrm{I}}+\mathbf{G}_{\mathbf{1 2}}$. (d) Sketches of samples MGC03 and MGC04 for which we resolve the MGC mode at $\mathbf{k}_{\mathrm{I}}+\mathbf{G}_{\mathbf{1 2}}$ in (e) reflection $(\Delta S 11)$. (f) In transmission geometry the signals of the mode at $\mathbf{k}_{\mathrm{I}}+\mathbf{G}_{\mathbf{1 2}}$ were below the relevant noise levels. Note that samples displayed in (d) lack the nanomagnet array underneath the detector CPW. In (e) we use arrows to indicate the position of the calculated branch. The microwave power amounted to $0 \mathrm{dBm}$. For fields above $60 \mathrm{mT}$, spectra were consistent with linear response. Vertical lines in (b) and (e) indicate the frequencies and field at which the line plots of (c) and (f) were extracted. In MGC03 we encountered a larger noise level than in the other devices. 
data for $\theta=0$ deg taken both in reflection (linear magnitude of $\Delta S 11$ ) and transmission measurements (imaginary part of $\Delta S 21$ ). The arrangement and array sizes of nanomagnet arrays varied from sample to sample (Tab. I). For example, MGC01 and MGC02 contained nanodisks underneath both the emitter and detector CPWs. Contrary to this, samples MGC03 and MGC04 had a nanomagnet array only underneath the emitter CPW. In all spectra we resolve resonances at $k_{\mathrm{I}}$ and larger wave vectors excited directly by the CPW (indicated by yellow arrows). SW excitations highlighted by blue arrows in Fig. 5 (b) are attributed to the MGC effect. The dashed red lines in the spectra represent the calculated eigenfrequencies of a SW with $k_{\mathrm{I}}+G_{12}{ }^{27}$. Corresponding resonances were resolved in the propagation signals $\Delta S 21$ of Fig. 5 (c), i.e., in both devices with symmetrically designed emitter and detector nanomagnet arrays. Note that sample MGC02 represents a bicomponent magnonic crystal which extends from the emitter to the detector CPW and most likely modifies the band structure of YIG between the CPWs. Strikingly, in $\Delta S 21$ of Fig. 5 (f) we do not resolve a mode close to the calculated branch $k_{\mathrm{I}}+G_{12}$. In MGC03 and MGC04, the detector CPWs do not contain nanomagnets. Particularly, the line spectrum of MGC04 exhibits the same small noise level as MGC01 but does not display the strong oscillations seen in Fig. 5 (c).

We now discuss our observations and results in detail. In our samples a propagation signal consistent with the [01] direction $(\delta=0 \mathrm{deg})$ has not been resolved. Note that $s_{\text {eff }}$ approaches $\infty$ for $\delta=0$ deg, i.e., $s_{\text {eff }}$ is larger than the decay length ${ }^{20}$. Accordingly, amplitudes of MGC SWs emitted along [01] direction are expected to be too small to induce relevant voltages in a detector CPW which is collinear with the emitter CPW. Reciprocal lattice vectors indicated by green squares in Fig. 2 are ambiguous. The bare CPW is known to emit similar wave vectors $|\mathbf{k}|$ by itself ${ }^{9}$. Transmitted signals might reflect both MGC modes and excitation related to high-indexed Fourier components of the CPW's radiofrequency field ${ }^{9}$. MGC modes unambiguously identified in Fig. 3 (a) are highlighted in Fig. 2 in yellow color without a green border. MGC modes resolved additionally in propagation at $\theta=0$ deg [Fig. 3 (b)] are depicted in purple color. These propagating MGC modes are observed mainly around the high symmetry directions [10] and [11] in reciprocal space [Fig. 2]. High-frequency MGC modes in YIG exhibit an eigenfrequency that overlaps with the resonance frequency of the Py nanodisks. In this case the coupling between the electromagnetic wave in the CPW and the SW in YIG is enhanced by the dynamic susceptibility of the Py nanodisks. This type of SWs is known as the resonant MGC mode ${ }^{21}$. Note that for the detection of MGC modes the detector CPW needed to contain an appropriate nanomagnet array. When using a bare detector CPW we did not resolve a branch close to the calculated mode $k_{\mathrm{I}}+G_{12}$ (compare the right graph of Fig. 5 (f) where the noise level is particularly low). The MGC effect is operative for both emitter and detector CPWs. For detection it is complementary to the thermoelectric and inverse spin Hall effects that were used to measure spin waves down to $\lambda=125 \mathrm{~nm}$ and did not provide access to the phase of detected spin waves ${ }^{33,34}$. In our experiments we performed phase-sensitive detection of spin waves. The phase is a key parameter for magnetologic based on spin waves ${ }^{35}$. Importantly, the YIG film used in our studies was thicker by about an order of magnitude compared to the earlier investigations on MGC modes ${ }^{20,21}$. The results presented here substantiate the versatility of the grating coupler effect for the generation and detection of short-waved magnons in general and also in commercial YIG films exhibiting a large thickness.

In conclusion we have reported MGC-excited spin waves propagating into different lateral directions of a ferrimagnetic thin film using a single CPW. An MGC mode consistent with a wavelength of $111 \mathrm{~nm}$ was detected over an effective distance of $77 \mu \mathrm{m}$ using emitter and detector CPWs with symmetrically designed nanomagnet arrays. The multi-directional emission and detection via MGCs is advantageous when designing multi-branched networks of integrated magnonic circuits that are interfaced with conventional microwave antenna.

Added note In the course of the review process we became aware of Ref. [36] in which a one-dimensional grating coupler was used to generate short-waved magnons propagating over a long distance of $60 \mu \mathrm{m}$ with $\delta=90 \mathrm{deg}$. Our results are consistent in that we use a two-dimensional grating coupler and emit short-waved magnons propagating in further directions on the chip with $\delta \neq 90$ deg over a long distance of up to effectively $86 \mu \mathrm{m}$. We acknowledge support by the DFG via the Cluster of Excellence "Nanosystems Initiative 
Munich NIM-2" and GR1640/5-2.

${ }^{1}$ A. Khitun, M. Bao, and K. Wang, J. Phys. D: Appl. Phys. 43, 264005 (2010).

${ }^{2}$ J. A. Davis, R. Venkatesan, A. Kaloyeros, M. Beylansky, S. J. Souri, K. Banerjee, K. C. Saraswat, A. Rahman, R. Reif, and J. D. Meindl, Proc. IEEE 89, 305 (2001).

${ }^{3}$ B. Heinrich, C. Burrowes, E. Montoya, B. Kardasz, E. Girt, Y. Song, Y. Sun, and M. Wu, Phys. Rev. Lett. 107, 066604 (2011).

${ }^{4}$ Y. Sun, Y. Song, H. Chang, M. Kabatek, M. Jantz, W. Schneider, M. Wu, H. Schultheiss, and A. Hoffmann, Appl. Phys. Lett. 101, 152405 (2012).

${ }^{5}$ V. Castel, N. Vlietstra, B. van Wees, and J. Youssef, Phys. Rev. B 86, 134419 (2012).

${ }^{6}$ O. d'Allivy Kelly, A. Anane, R. Bernard, J. Youssef, C. Hahn, A. H. Molpeceres, C. Carrétéro, E. Jacquet, C. Deranlot, P. Bortolotti et al., Appl. Phys. Lett. 103, 082408 (2013).

${ }^{7}$ H. Yu, O. d'Allivy Kelly, V. Cros, R. Bernard, P. Bortolotti, A. Anane, F. Brandl, R. Huber, I. Stasinopoulos, and D. Grundler, Sci. Rep. 4, 6848 (2014).

${ }^{8}$ C. Hauser, T. Richter, N. Homonnay, C. Eisenschmidt, M. Qaid, H. Deniz, D. Hesse, M. Sawicki, S. Ebbinghaus, and G. Schmidt, Sci. Rep. 6, 20827 (2016).

${ }^{9}$ S. Maendl, I. Stasinopoulos, and D. Grundler, Appl. Phys. Lett. 111, 012403 (2017).

${ }^{10}$ L. Lutsev, A. Korovin, V. Bursian, S. Gastev, V. Fedorov, S. Suturin, and N. Sokolov, Appl. Phys. Lett. 108, 182402 (2016).

${ }^{11}$ K. S. Lee and S. K. Kim, J. Appl. Phys. 104, 053909 (2008).

${ }^{12}$ S. Vasiliev, V. Kruglyak, M. Sokolovskii, and A. Kuchko, J. Appl. Phys. 101, 113919 (2007).

${ }^{13}$ F. Gertz, A. Kozhevnikov, Y. Filimonov, and A. Khitun, IEEE Trans. Magn. 51, 4002905 (2015).

${ }^{14}$ A. Chumak, V. Vasyuchka, A. Serga, and B. Hillebrands, Nat. Phys. 11, 453 (2015).

${ }^{15}$ M. Eshaghian-Wilner, A. Khitun, and K. Wang, US Patent 8,193,598, B2 (2012).

${ }^{16}$ A. Khitun, M. Bao, and K. Wang, IEEE Trans. Magn. 44, 2141 (2008).

${ }^{17}$ C. W. Sandweg, Y. Kajiwara, A. V. Chumak, A. A. Serga, V. I. Vasyuchka, M. B. Jungfleisch, E. Saitoh, and B. Hillebrands, Phys. Rev. Lett. 106, 216601 (2011).

${ }^{18}$ M. Madami, S. Bonetti, G. Consolo, S. Tacchi, G. Carlotti, G. Gubbiotti, F. B. Mancoff, M. A. Yar, and J. Akerman, Nat. Nanotechn. 6, 635 (2011).

${ }^{19}$ S. Wintz, V. Tyberkevych, M. Weigand, J. Raabe, J. Lindner, A. Erbe, A. Slavin, and J. Fassbender, Nat. Nanotechn. 11, 948 (2016).

${ }^{20}$ H. Yu, G. Duerr, R. Huber, M. Bahr, T. Schwarze, F. Brandl, and D. Grundler, Nat. Commun. 4, 3702 (2013).

${ }^{21}$ H. Yu, O. d'Allivy Kelly, V. Cros, R. Bernard, P. Bortolotti, A. Anane, F. Brandl, F. Heimbach, and D. Grundler, Nat. Commun. 7, 11255 (2016).

${ }^{22}$ C. Dubs, O. Surzhenko, R. Linke, A. Danilewsky, U. Brückner, and J. Dellith, J. Phys. D: Appl. Phys. 50, 204005 (2017).

${ }^{23}$ Matesy GmbH, Otto-Schott-Str. 13, 07745 Jena, Germany.

${ }^{24}$ G. Duerr, M. Madami, S. Neusser, S. Tacchi, G. Gubbiotti, G. Carlotti, and D. Grundler, Appl. Phys. Lett. 99, 202502 (2011).

${ }^{25}$ G. Counil, J. Kim, T. Devolder, C. Chappert, K. Shigeto, and Y. Otani, J. Appl. Phys. 95, 5646 (2004).

${ }^{26}$ S. Neusser, G. Duerr, H. Bauer, S. Tacchi, M. Madami, G. Woltersdorf, G. Gubbiotti, C. Back, and D. Grundler, Phys. Rev. Lett. 105, 067208 (2010).

${ }^{27}$ B. Kalinikos and A. Slavin, J. Phys. C: Sol. St. Phys. 19, 7013 (1986).

${ }^{28}$ J. Kunzler, L. Walker, and J. Galt, Phys. Rev. 119, 1609 (1960).

${ }^{29}$ D. T. Edmonds and R. G. Petersen, Phys. Rev. Lett. 2, 499 (1959).

${ }^{30}$ A. Gurevich and G. Melkov, Magnetization Oscillations and Waves (CRC Press, 1996).

${ }^{31}$ F. Heimbach, T. Stückler, H. Yu, and W. Zhao, J. Magn. Magn. Mater. 450, 29 (2018).

${ }^{32}$ M. Krawczyk, S. Mamica, M. Mruczkiewicz, J. W. Klos, S. Tacchi, M. Madami, G. Gubbiotti, G. Duerr, and D. Grundler, J. Phys. D: Appl. Phys. 46, 495003 (2013).

${ }^{33}$ H. Schultheiss, J. E. Pearson, S. D. Bader, and A. Hoffmann, Phys. Rev. Lett. 109, 237204 (2012).

${ }^{34}$ T. Braecher, M. Fabre, T. Meyer, T. Fischer, S. Auffret, O. Boulle, U. Ebels, P. Pirro, and G. Gaudin, Nano Lett. 17, 7234 (2017).

${ }^{35}$ R. Hertel, W. Wulfhekel, and J. Kirschner, Phys. Rev. Lett. 93, 257202 (2004).

${ }^{36}$ C. Liu, J. Chen, T. Liu, F. Heimbach, H. Yu, Y. Xiao, J. Hu, M. Liu, H. Chang, T. Stueckler et al., Nat. Commun. 9, 738 (2018). 


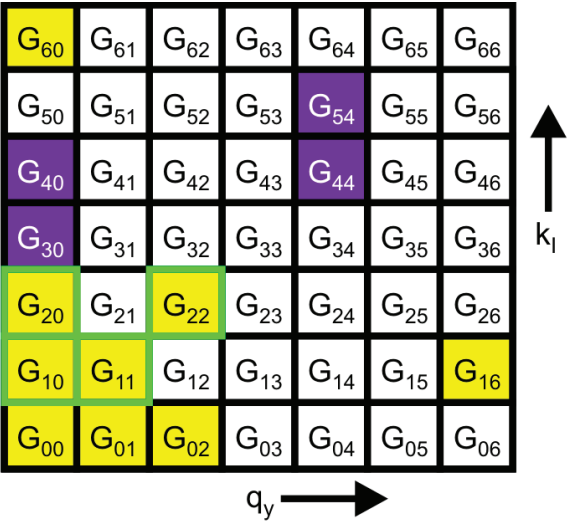




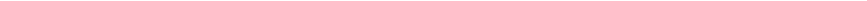


